Research Paper

\title{
A combination of surgery, theranostics, and liquid biopsy - a personalised oncologic approach to treatment of patients with advanced metastatic neuroendocrine neoplasms
}

\author{
Andrea Frilling ${ }^{1 凶}$, Ashley K. Clift ${ }^{1}$, Adam E. Frampton ${ }^{1}$, Jamshed Bomanji² ${ }^{2}$, Daniel Kaemmerer ${ }^{3}$, Adil \\ Al-Nahhas ${ }^{4}$, Ali Alsafi ${ }^{4}$, Mark Kidd ${ }^{5}$, Irvin M. Modlin', Dieter Hoersch ${ }^{7}$ and Richard P. Baum ${ }^{8}$ \\ 1. Department of Surgery and Cancer, Imperial College London, London, UK. \\ 2. Department of Nuclear Medicine, University College London Hospitals, London, UK. \\ 3. Department of General and Visceral Surgery, Zentralklinik Bad Berka, Bad Berka, Germany. \\ 4. Department of Imaging and Nuclear Medicine, Imperial College London, London, UK. \\ 5. WREN Laboratories, Branford, USA. \\ 6. Gastroenterological and Endoscopic Surgery, Yale University School of Medicine, New Haven, USA. \\ 7. Department of Gastroenterology/Endocrinology, Zentralklinik Bad Berka, Bad Berka, Germany. \\ 8. CURANOSTICUM Wiesbaden-Frankfurt at DKD Helios Klinik, Wiesbaden, Germany. \\ $\square$ Corresponding author: Professor Andrea Frilling, Department of Surgery and Cancer, Imperial College London, London, UK. Email: a.frilling@imperial.ac.uk
} (c) The author(s). This is an open access article distributed under the terms of the Creative Commons Attribution License (https://creativecommons.org/licenses/by/4.0/). See http:/ /ivyspring.com/terms for full terms and conditions.

Received: 2020.08.11; Accepted: 2021.01.14; Published: 2021.03.19

\begin{abstract}
Rationale: Neuroendocrine neoplasia (NEN) of small bowel (SBNEN) frequently present with metastatic disease. Theranostics (molecular imaging followed by targeting therapy) allow for personalised medicine. Liquid biopsies enable precise identification of residual disease and real-time monitoring of therapeutic response. Our aim was to determine the clinical utility of a combination of surgery, theranostics, and a multigene blood measurement in metastasised SBNEN.

Methods: Inclusion criteria were SBNEN, G1/G2 NEN, initial tumour diagnosis, stage IV NEN, positivity on ${ }^{68} \mathrm{Ga}$ somatostatin analogue PET/CT, eligible for surgery, and $177 \mathrm{Lu}$ peptide receptor radionuclide therapy (PRRT). Blood samples for NETest were collected longitudinally. Progression-free survival (PFS) and overall survival (OS) were calculated. NETest results were assessed prior to surgery and during clinical follow-up.

Results: A surgical cohort of 39 SBNEN patients met eligibility criteria. Thirty-two patients underwent ileal resection and 7 right hemicolectomy. The mean number of ${ }^{177} \mathrm{Lu}$ PRRT cycles was 4 . Mortality was nil. Surgical morbidity was $10.3 \%$. Transient grade $1 / 2$ toxicity occurred in $41 \%$ (PRRT). NETest scores ( $n=9$ patients) decreased in $100 \%$ following treatment and correlated with diminished tumour volume and disease stabilization following surgery and PRRT. Median follow-up: 78 months. Median PFS and OS: 42.7 and 110 months, respectively. Progression-free survival at 1-, 3-, and 5-years was $79.4 \%, 57.1 \%$ and $40.5 \%$, respectively. Overall survival at 1-, 3-, and 5-years was $97.4 \%, 97.4 \%$, and $94.1 \%$, respectively.

Conclusions: Surgery combined with ${ }^{177 L u}$ PRRT is safe and provides favourable PFS and OS in selected patients with advanced SBNEN. Liquid biopsy (NETest) has the potential to accurately delineate disease status.
\end{abstract}

Key words: Neuroendocrine neoplasia, small bowel, surgery, peptide receptor radionuclide therapy, NETest, mRNA, multianalyte gene biomarker.

\section{Introduction}

Patients with well-differentiated, grade $(G) 1$ and G2 entero-pancreatic neuroendocrine neoplasms $(\mathrm{NEN})$ are frequently metastatic at initial diagnosis. Metastases are detectable in $45 \%-90 \%$ of patients with
NEN originating from small bowel (SBNEN) [1, 2] and in $40 \%-80 \%$ of pancreatic NEN (PanNEN) [3]. First line treatment is provided by radical surgery to eliminate the primary tumour including loco-regional 
lymph node metastases and liver metastases and provides the best long-term outcome $[4,5]$.

A substantial number of SBNEN patients and those with NEN originating from the pancreas are diagnosed with resectable primary tumours in the presence of distant metastases not amenable to conventional surgical resection. There is clear indication for resection of the primary tumour in patients who are symptomatic due to functional syndromes and/or loco-regional tumour-related symptoms regardless of the disease stage. However, there is controversy regarding benefit of resection of asymptomatic SBNEN or PanNEN in the presence of unresectable liver metastases [6-8]. In the absence of randomised controlled trials, a systematic review showed an improved pooled 5-year overall survival of $73.1 \%$ (range $57-81 \%$ ) for patients with resected SBNEN and unresectable liver metastases compared to $36.6 \%$ (range $21 \%-46 \%$ ) in the unresected group [9]. Recently, a number of effective targeted treatment options have been introduced into the palliative management of patients with metastasised, non-resectable NEN. These include long-acting repeatable (LAR) somatostatin analogues, everolimus, sunitinib, and systemic peptide receptor radionuclide therapy (PRRT) with Yttrium-90 ( $\left.{ }^{90} \mathrm{Y}\right)$-DOTA0 Try ${ }^{3}$ octreotide (90Y-DOTATOC) [10] or with Lutetium-177 $\left({ }^{177} \mathrm{Lu}\right)$-DOTA ${ }^{0}-$ Try $^{3}$-octreotate $\left({ }^{177} \mathrm{Lu}-\right.$ DOTATATE) or ${ }^{177} \mathrm{Lu}$-DOTATOC $[11,28]$. Thus, a promising, novel approach of evolving interest has been the introduction of multimodal therapy combining surgery with patient-tailored systemic therapies targeting non-resectable disease.

'Theranostics' refers to the use of molecular targeting vectors labelled with radionuclides which can be used for both diagnostic imaging and tumour-specific therapy [12, 13]. Neuroendocrine neoplasms represent an ideal condition for theranostic approaches as most well- and moderatelydifferentiated NEN overexpress somatostatin receptors (SSTR) suitable for targeting with radiolabelled peptides for both diagnostic and therapeutic purposes [12,14]. First, the tumour is visualised with ${ }^{68}$ Gallium $\left({ }^{68} \mathrm{Ga}\right)$ labelled DOTA-peptides with high binding affinity to somatostatin receptors utilising the positron emission (PET) / computed tomography (CT) (PET/CT) [15]. Second, DOTA-peptides radiolabelled with a therapeutic radionuclide such as ${ }^{177} \mathrm{Lu}$ or ${ }^{90} \mathrm{Y}$ are applied for therapeutic purpose. The efficacy of PRRT in terms of favourable progression-free survival (PFS) and overall survival (OS) in NEN patients compared to historical controls has been shown in numerous phase I and phase II studies [16-19], in larger patient cohorts $[20,21]$ and in the phase III trial of
${ }^{177} \mathrm{Lu}$-DOTATATE in patients with advanced well-differentiated midgut NEN with disease progression during first-line somatostatin analogue therapy (NETTER-1 trial, NCT 01578239) [11]. In the ${ }^{177} \mathrm{Lu}$-DOTATATE group, PFS at month 20 was $65.2 \%$ (95\% confidence interval $[\mathrm{CI}], 50.0$ to 76.8 ) compared to $10.8 \%$ (95\% CI, 3.5 to 23.0 ) in the control group. A meta-analysis on efficacy of ${ }^{177} \mathrm{Lu}$ PRRT in patients with unresectable metastasised NEN demonstrated a pooled disease response rate of $29.1 \%(95 \%$ CI: $20.2 \%-38.9 \%$ ), and disease control rate of $74.1 \%$ (95\% CI: $67.8 \%-80.0 \%$ ) [22].

Liquid biopsies have garnered rising interest over the last few years given their potential for longitudinal, real-time status assessment and provision of diagnostic, prognostic, and predictive markers for precision medicine [23, 24]. A blood-based 51-gene multianalyte transcript biomarker has been developed specifically for well-differentiated NEN to dynamically characterise tumour behaviour from genomic information [25]. The test (NETest) is $>90 \%$ accurate in NEN originating from the gastro-entero-pancreatic and bronchopulmonary system [26-28]. Its main clinical aim is to monitor disease progress, predict therapeutic efficacy and assess prognosis. In addition, it can be used to delineate the effectiveness of operative resection and ablation strategies and identify minimal residual disease [29, 30]. In direct comparative studies it outperforms the current standard tumour marker chromogranin [25]. The sensitivity and specificity of the NETest is $>95 \%$ and $>90 \%$, respectively [31]. In a US Registry-based study, NETest diagnostic accuracy was $96 \%$ and concordant with image-demonstrable disease in 95\% (NCT 02270567) [28]. High NETest score correlated with progressive disease and low NETest score with stable disease.

In this study, we aimed to evaluate the outcome of a multimodal approach combining surgery and theranostics in well-characterised group with metastatic small bowel neuroendocrine neoplasms. In addition, we investigated whether a multianalyte gene transcript biomarker could assess disease status and delineate disease reduction following multimodal treatment.

\section{Patients and Methods}

Consecutive patients with small bowel NEN (SBNEN) treated between 01 January 2007 and 31 December 2018 at Imperial College Health Care NHS Trust (ICHCNT), London, UK and at the Zentralklinik Bad Berka (ZKBB), Bad Berka, Germany, respectively were considered in this longitudinal study. Both institutions, which are Centres of Excellence of the European Neuroendocrine Tumour Society, followed 
the same diagnostic and treatment protocols. All patients were discussed in local multidisciplinary team meetings. Clinical information was prospectively collected in institutional NEN databases.

Inclusion criteria were well- or moderately differentiated, grade (G) 1 and G2 NEN originating from small intestine, no previous treatment for NEN apart from injections of high-dose octreotide LAR for amelioration of hormonal symptoms associated with carcinoid syndrome, symptoms caused by loco-regional tumour growth, unresectable stage IV disease, positivity on ${ }^{68} \mathrm{Ga}-\mathrm{DOTA}-\mathrm{D}-\mathrm{Phe1}-\mathrm{Ty} 3 \mathrm{-}$ octreotide (DOTATOC) PET/CT, ${ }^{68} \mathrm{Ga}$-DOTA-1-Nal3octreotide (DOTANOC) PET/CT, and ${ }^{68} \mathrm{Ga}-$ DOTA-D-Phe1-Tyr3-Thr8-octreotide (DOTATATE) $\mathrm{PET} / \mathrm{CT}$, respectively (Figure 1), absence of severe carcinoid heart disease, eligibility for surgery within four weeks following initial diagnosis, and eligibility for ${ }^{177} \mathrm{Lu}$ PRRT within three months following surgery. Patients who had symptoms of carcinoid syndrome and were referred to us having not yet started treatment for such symptoms were started on octreotide LAR. The treatment was discontinued prior to PRRT with a withdrawal period of 4-6 weeks. Inclusion of patients at ICHCNT was impacted by limited availability of PRRT in UK during the study period. Resectability of liver metastases was assessed with MRI and SSTR-PET/CT. Follow-up encompassed standard biochemistry, morphologic imaging every 3-6 months, SSTR-PET/CT based imaging every 6-12 months, and in a subgroup of patients, blood sampling for NETest prior to surgery and at 6 months after the last PRRT cycle. Overall efficacy of treatment and response to PRRT were assessed with SSTR-PET/CT at 6 months after the last treatment cycle. Outcome measures included 1-, 3-, and 5-year progression-free (PFS) and overall survival (OS) from initial diagnosis. Only patients with a follow-up of at least 6 months were considered.

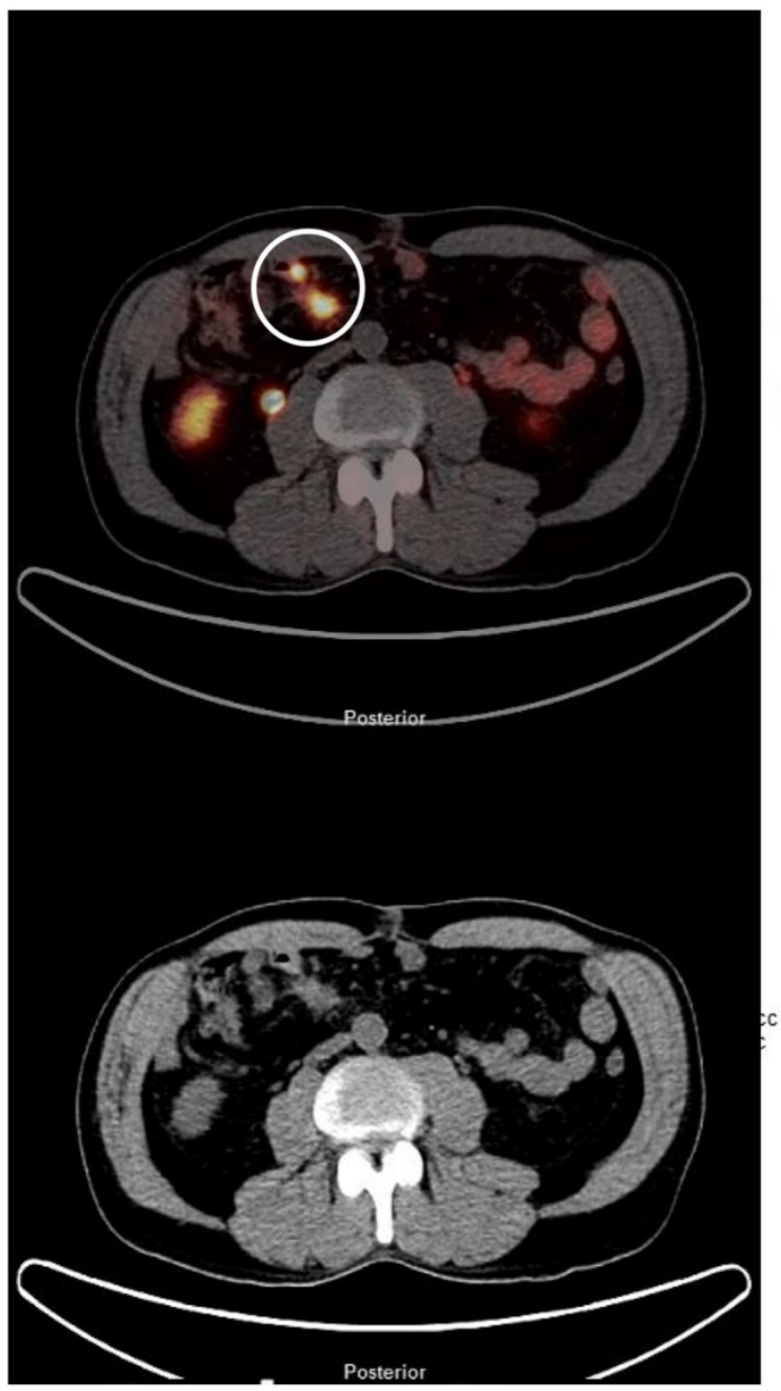

Figure $1 .{ }^{68} \mathrm{Ga}$ DOTATATE PET/CT demonstrating a SBNEN with lymph node metastases and distant metastases. The primary tumour and locoregional lymph node metastases (stage I) are marked with a circle on the fused image (left side) and on the PET component (right side). The patient also had multiple bone metastases (rib, cervical spine and thoracic spine [arrows]) and liver metastases. 
SSTR-PET/CT was performed according to standard protocols as described previously $[32,33]$. The criteria used to define eligibility for PRRT were in accordance with published guidelines for PRRT and included a Karnofsky index of $>60 \%$, life expectancy of more than 6 months, somatostatin receptor positive NEN and adequate bone and renal function [33]. In short, $7.4 \mathrm{GBq}(200 \mathrm{mCi})$ of ${ }^{177} \mathrm{Lu}-\mathrm{DOTATATE}$ (Lutathera $\left.{ }^{\circledR}\right)$ (ICHCNT) and ${ }^{177} \mathrm{Lu}$-DOTATOC with a mean applied activity of $6.54 \mathrm{GBq}$ (ZKBB) was infused intravenously over a period of 15-30 minutes. Most patients received three to four infusions every eight to 12 weeks. An intravenous reno-protective amino acid solution was administered prior to the administration of the therapeutic dose and continued for four hours thereafter [33]. Individual therapy planning based on pre-therapy SSTR-PET/CT as the dosimetry protocol and SPECT/CT studies acquired at $24 \mathrm{~h}$ after each PRRT cycle were considered. With individual dosimetry we aimed to establish personalised treatment doses to ensure that the total renal dose and the bone marrow dose did not exceed the $23 \mathrm{~Gy}$ and the 2 Gy limit, respectively.

Regular blood tests for assessment of adverse events and imaging were carried out. Final restaging and assessment of response to treatment were performed with SSTR-PET/CT at 6 months after the last PRRT cycle. Responses were evaluated with European Organisation for Research and Therapy of Cancer (EORTC) criteria [34] (PET component of $\mathrm{PET} / \mathrm{CT}$ ) as well as by Response Evaluation Criteria in Solid Tumors (RECIST) [35] (CT component of PET/CT or MRI). Adverse events were assessed from laboratory data at the time of occurrence and graded according to the Common Terminology Criteria for Adverse Events (CTCAE) version 4.0 (NCI, Bethesda, USA).

Our standard surgical approach included systematic palpation of the entire small bowel starting at the Treitz ligament and ending at the caecal valve, assessment of mesenteric lymph node metastases involvement (stage I-IV) [36], and segmental small bowel resection/s including mesenteric loco-regional disease. A lymph node-first, intestinal-sparing principle was followed. For tumours localized in the terminal ileum, concomitant oncologic right hemicolectomy was performed. All patients with liver metastases and / or carcinoid syndrome were treated with octreotide (50 microgram/hour intravenously) for 12 hours prior to surgery and $24 \mathrm{~h}$ thereafter. Resected specimens were subjected to immuno-histochemical examination including assessment of Ki67\% for tumour grading. Surgical morbidity was assessed according to Clavien-Dindo classification [37] and mortality recorded.

\section{Multigene blood analysis}

Only patients seen at ICHCNT and treated between 2014 and 2018 were considered as NETest candidates for logistic reasons. In a sub-cohort of patients (SBNEN, $n=9$ ), blood samples for the NETest were collected prior to surgery and at 6 months following the last PRRT cycle. All patients considered for NETest completed blood sampling according to the protocol. The analysis comprised a 2-step protocol (RNA isolation/cDNA production and $\mathrm{qPCR}$ ) from EDTA-collected whole blood [25]. Transcripts (mRNA) were isolated from EDTA-collected whole blood samples (mini blood kit, Qiagen, Valencia CA) and real-time PCR performed on pre-spotted plates. Target transcript levels were normalized and quantified versus a population control. Results were expressed as an activity index (NETest score) from 0-100 ('normal' score range is 0-20). Assays were undertaken using de-identified samples in a central USA clinically and federally certified laboratory (Wren Laboratories CL-0704, CLIA 07D2081388). NETest results were correlated with clinical follow-up data. The study was approved by the Imperial College Healthcare Tissue Bank Committee and a National Research Ethics Committee (07/MRE09/54). Informed consent was obtained from all patients.

\section{Statistical analysis}

Patient demographics and tumour characteristics were reported with descriptive statistics. Progression-free survival and OS were recorded by using Kaplan-Meier methodology and were calculated from initial diagnosis to the date of disease progression evident on imaging and the date of death or last-follow up visit, respectively. Mann-Whitney U-test was used to evaluate changes in NETest. A $p$ value of $<0.05$ was deemed statistically significant. Stata v15 was used for statistical analyses.

\section{Results}

\section{Surgery}

Thirty-nine SBNEN patients (24 males $(61.5 \%)$ median age at surgery 58.8 years [range 32.1-78.4]) were enrolled prospectively (Table 1). During the study period, 395 SBNEN patients were treated in total. All patients were symptomatic due to local mass effect and/or hormonal excess. In all patients, anatomic imaging and/or SSTR-PET/CT revealed distant metastases not suitable for resection. Of the SBNEN patients, 32 (82.1\%) underwent ileal resection(s) and mesenteric lymphadenectomy and in $7(17.9 \%)$, concomitant oncologic right hemicolectomy was carried out. Twelve patients $(30.8 \%)$ had multifocal tumours (mT1-T3). The majority of patients 
with unifocal primary tumours had T2 and T3 lesions, respectively $(88.9 \%)$. Mesenteric lymph node metastases were evident in $37 / 39$ patients $(94.9 \%)$ and resected in 35/37 (94.6\%) (Figure 2). In two patients with stage IV lymph node metastases and liver metastases, resection of the metastatic lymph node bulk was abandoned to avoid the risk of bowel ischemia and short bowel syndrome. In all patients, surgery took place within 4 weeks following initial diagnosis. All 35 patients with liver metastases $(89.7 \%)$ had type II (isolated metastatic bulk accompanied by smaller deposits), or type III (disseminated metastatic spread) liver metastases with $>75 \%$ liver parenchyma having evidence of metastatic involvement. Even under consideration of the $\leq 70 \%$ rule for debulking of NEN liver metastases, down-staging procedures, and two-stage hepatectomies, they were considered as non-resectable.

Table 1. Clinical and pathological characteristics of the study cohort $(n=39)$.

\begin{tabular}{ll}
\hline Characteristic & Number of patients (\%) \\
\hline Sex & \\
Male & $24(61.5)$ \\
Female & $15(38.5)$ \\
Age, median (range) & 58.8 years (32 to 78 years) \\
Primary tumour site & \\
Small bowel & $39(100)$ \\
Tumor focality & \\
Unifocal & $27(69.2)$ \\
Multifocal & $12(30.7)$ \\
Symptoms due to mass effect & \\
Pain & $39(100)$ \\
Bleeding & $15(38.5)$ \\
Intestinal obstruction & $6(15.4)$ \\
Functional symptoms & \\
Carcinoid syndrome & $25(64.1)$ \\
Surgical procedures (small bowel) & \\
Segmental resection/s & $32(82.1)$ \\
Right hemicolectomy & $7(17.9)$ \\
Tumour grade & \\
Grade 1 & $30(76.9)$ \\
Grade 2 & $9(23.1)$ \\
Grade 3 & $0(0)$ \\
Tumour stage & $16(41)$ \\
T1 & $10(25.1)$ \\
T2 & $37(7.7)$ \\
T4 & $12(30.8)$ \\
mT1-T3 & \\
Lymph node metastases & \\
Distant metastases & \\
Liver & \\
Bone & \\
Other & \\
\hline
\end{tabular}

$\mathrm{mT}=$ multifocal tumors.
The 90 -days postoperative morbidity was $10.3 \%$ (4/39) (all grade 1). The 90-days postoperative mortality was nil. The symptoms caused by loco-regional tumour extent recorded preoperatively diminished in all cases after surgery. Histology confirmed well-differentiated NEN grade 1 or grade 2 in all resected specimens.

\section{Lu PRRT}

Treatment was commenced in all 39 patients within 3 months following surgery. The mean number of ${ }^{177} \mathrm{Lu}$-octreotate cycles was 4 (range 2-6). The administered activity dose and the number of treatment cycles were determined with individual dosimetry. Transient grade $1 / 2$ toxicity occurred in 16 patients $(41 \%)$. There was no grade 3 toxicity. At 6 months following the last cycle, complete response, partial response and stable disease were seen in 3 $(7.69 \%), 25(64.1 \%)$, and $11(28.2 \%)$ patients, respectively. In the two patients with remaining stage IV lymph node metastases (both also had liver metastases), complete response and stable disease, respectively were achieved. No disease progression was recorded.

\section{Multigene blood analysis}

Nine patients were available for inclusion. The NETest was positive in $9 / 9(100 \%)$ patients prior to treatment and $8 / 9$ scores were $\geq 80$ (consistent with advanced disease, Figure 3). After treatment (surgery and PRRT), NETest scores dropped from $83 \pm 12$ to $34 \pm 15(p<0.0001)$ (Figure 3) concordant with imaging results in all 9 patients. Six exhibited scores $\leq 40$ (consistent with stable disease), 3 exhibited scores of 47-53. These scores preceded further therapy and disease status remained unchanged since the post-treatment NETest in all during further follow-up (median 18.5 months, range 6 to 46 months).

\section{Survival outcomes}

No patient was lost to follow-up. The median follow-up for the cohort was 78 months (range: 6 to 146 months), during which there were 13 deaths, all due to disease. Of all 39 patients, 26 developed progressive disease and required further treatment (not reported here). Median overall survival was 110 months (95\% CI: 80.8 to 138.7 months). Overall survival at 1-year, 3-years and 5-years was $97.4 \%$, $97.4 \%$ and $94.1 \%$, respectively. Median progression-free survival was 42.7 months (95\% CI: 24.7 to 72.4 months). Progression-free survival at 1 -year, 3-years and 5-years was $79.4 \%, 57.1 \%$, and $40.5 \%$, respectively (Figure 4 ). 


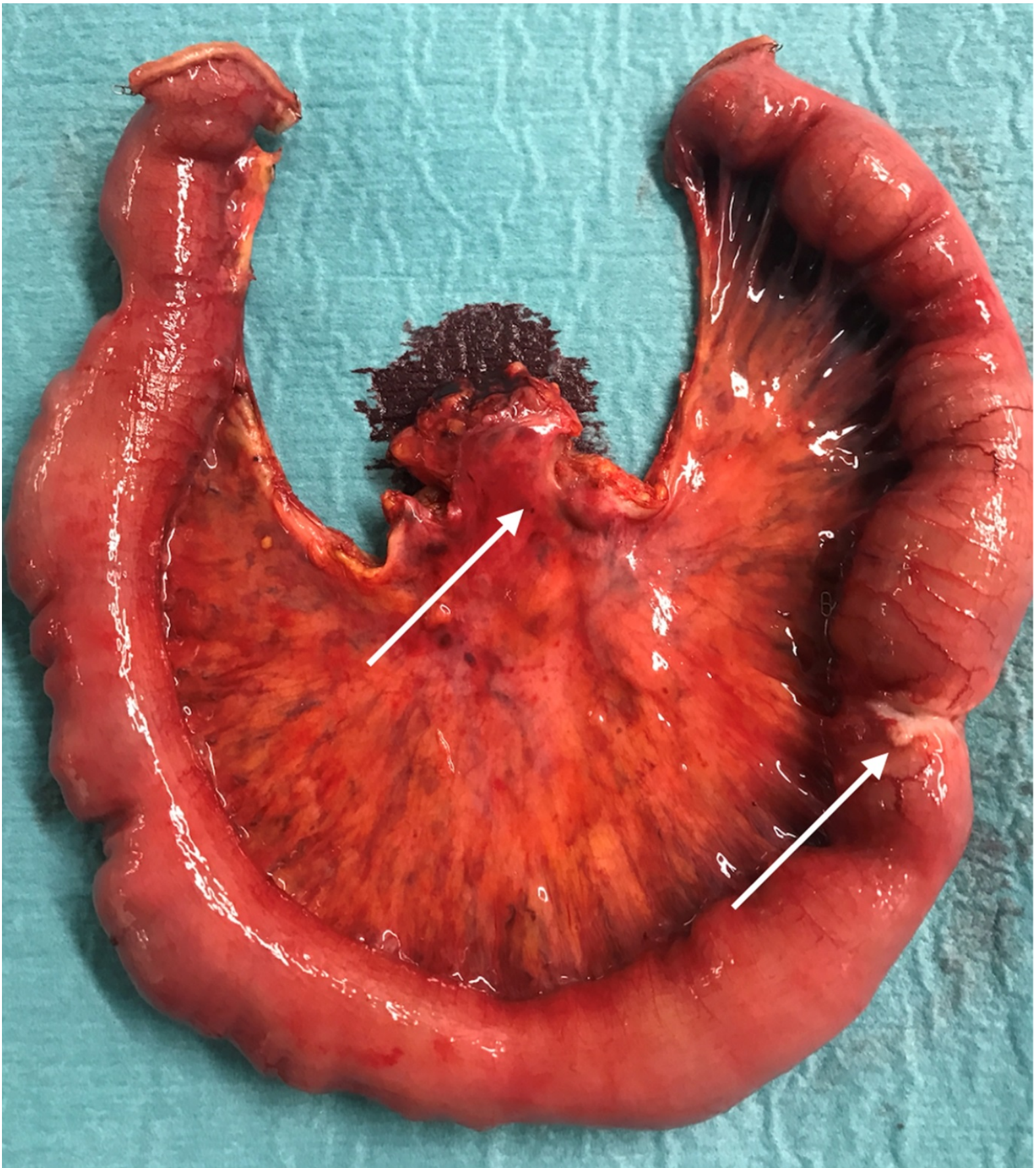

Figure 2. Surgical specimen of a patient with a SBNEN (arrow) causing intermittent bleeding and bowel occlusion. In addition, 11 further tumours all sized $\leq 5 \mathrm{~mm}$ and only detectable on palpation were found and confirmed as NEN on immunohistological examination. There were stage III mesenteric lymph node metastases (arrow).

\section{Discussion}

Small bowel NEN frequently present with synchronous distant metastases, undermining the efficacy of surgical treatment. Widespread use of SSTR-based PET/CT technology has improved detection of metastatic deposits and predicates upstaging of $30 \%-40 \%$ of NEN patients with only localised disease seen on standard imaging and change in treatment strategy in up to $45 \%$ by identifying lesions that are suitable for resection or detecting multiple unresectable lesions approachable by non-surgical therapeutic modalities [38-40]. Particularly challenging clinical scenarios include patients with NEN that are symptomatic due to local tumour effect since they require both surgery to eliminate the primary tumour and systemic treatment for metastases.

Thyroidectomy followed by ${ }^{131}$ Iodine (131I) radiotherapy for treatment of differentiated thyroid cancer accounts for an example par excellence of a multimodal treatment concept combining surgery for 
the primary tumour and theranostics for management of its metastatic and/or residual disease [41]. In NEN, combining surgery and targeted molecular radionuclide therapy (PRRT) has been applied mainly in neoadjuvant settings for down-staging of resectable or potentially resectable tumours [42-44], or as an upfront strategy to enhance response to PRRT and prolong survival [43, 45-47].

Our treatment strategy consisted of three steps; accurate staging with molecular imaging, surgery for elimination of loco-regional disease, and molecular therapy for targeting of unresectable metastases. With a median PFS of 42.73 months and OS at 5-years of $94.1 \%$ we have demonstrated in a group of stage IV SBNEN patients the efficacy of this approach. Surgical morbidity (all grade 1) of $10.3 \%$, and no relevant toxicity (all grade 1/2) of PRRT further underlined the justification of our concept. An overall response rate to PRRT of $71.8 \%$ achieved in this study confirmed favourable observations made by others treating specifically advanced midgut NEN with PRRT [11, 20]. We speculate that the favourable results achieved in our study may be related to manifold factors. Our patient cohort was very homogeneous (G1/G2 SBNEN only), our patients were relatively young (the median patient age was $<60$ years) and all patients underwent surgical treatment within 4 weeks after initial diagnosis, of which $>90 \%$ had radical resection of locoregional disease. Lastly, PRRT utilising personalised dosimetry was applied within 4 months of initial diagnosis, and was first-line therapy for non-resectable disease prior to progression in all patients.

Because of the limited efficacy of somatostatin analogues in patients with SBNEN and high hepatic tumour burden [48], the high success rate of therapy with 177Lu-octreotate when compared to somatostatin analogues [11] and the absence of serious side-effects, we and others [49] advocate its use in patients with SBNEN without waiting for tumour progression. In addition, it has been shown that the effect of PRRT negatively correlates with the volume of hepatic metastatic tumour burden.
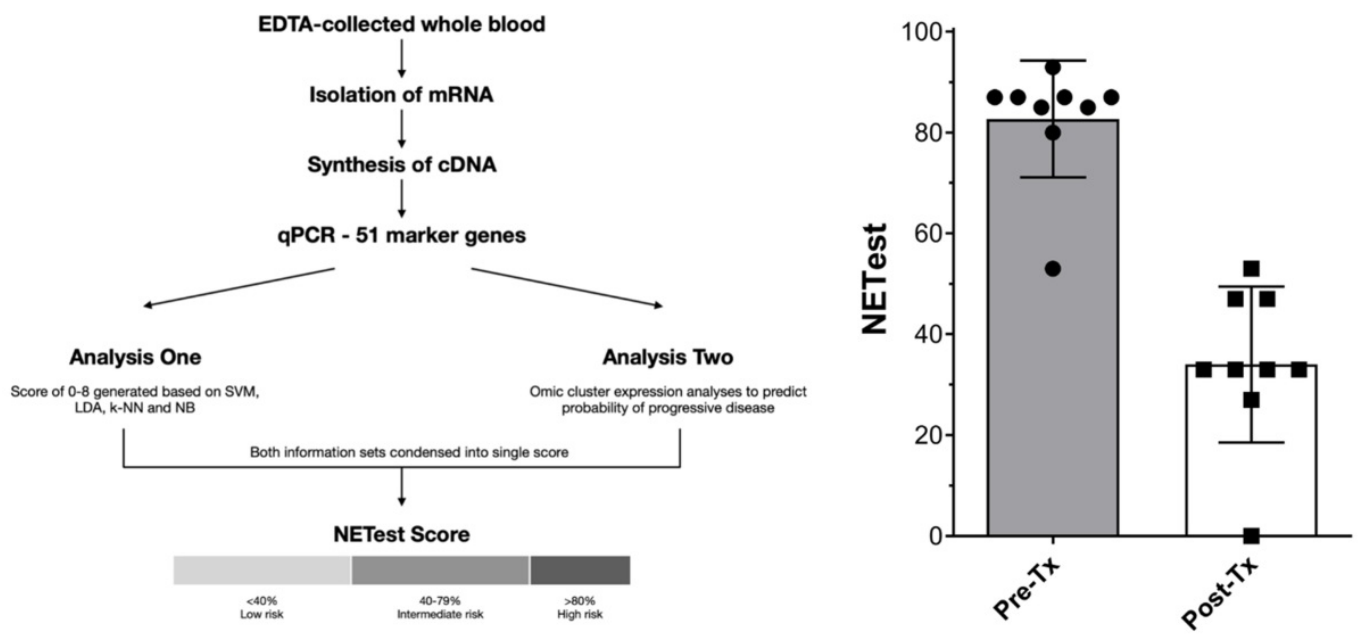

Figure 3. Representation of steps involved in NETest assay and derivation of results (3A, left) and NETest results pre-treatment and post-treatment (surgery followed by PRRT) for 9 patients in this study (3B, left).
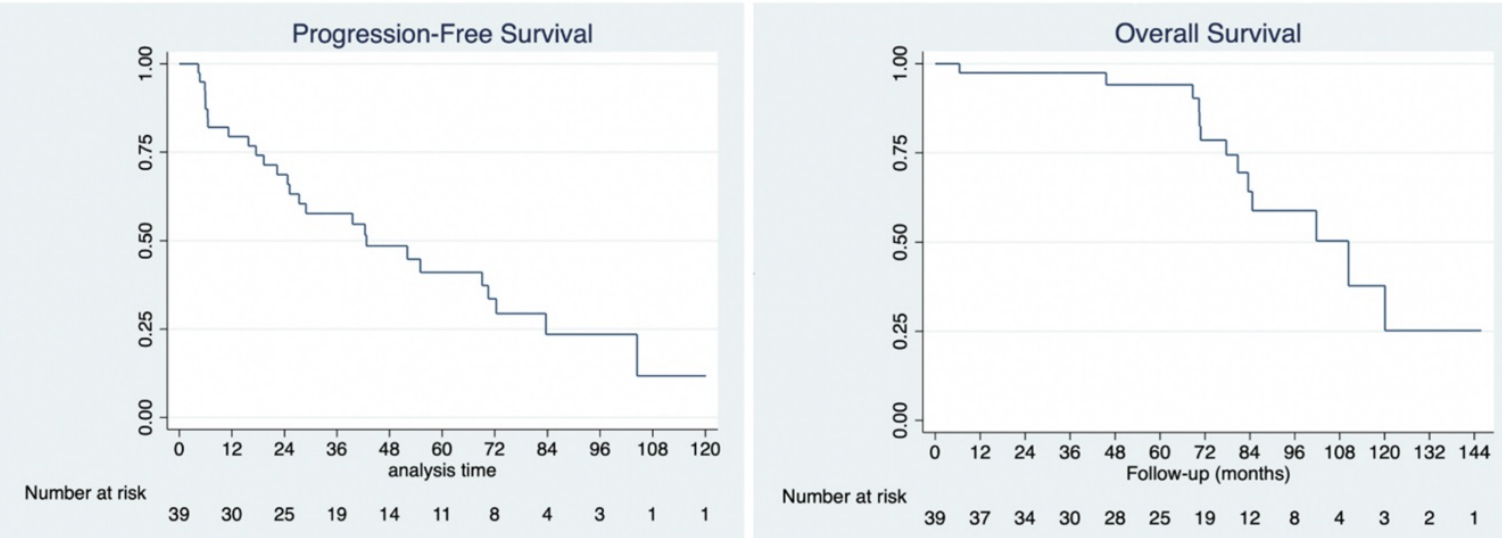

Figure 4. Kaplan-Meier analysis of progression-free survival (4A, left) and overall survival (4B, right). 
Surgery of SBNEN might be challenging due to two characteristic features of these tumours; primary tumour multifocality in up to $30 \%$ and mesenteric lymph node metastases frequently compromising major mesenteric vascularity (stage III) or encasing the mesenteric root (stage IV). Radical surgery has been shown to reduce the risk of local complications and increase long-term survival [1]. In this series, multifocal tumours were present in $30.8 \%$ and $>95 \%$ had mesenteric lymph node metastases of various stages. Although laparoscopic resection of SBNEN has been reported [50,51], we are in favour of laparotomy allowing for meticulous palpation of the entire small bowel and identification of frequently millimetre-sized intramural tumours not seen preoperatively on any imaging or endoscopy [52], and optimal extent of lymphadenectomy (at least 8 lymph nodes) under consideration of intestinal-sparing principles [53,54]. Hand-held gamma probe surgery using gallium-68-labeled somatostatin analogues has been shown to be a promising adjunct for real-time detection of small metastatic deposits and primary tumours $[55,56]$.

Personalized or precision medicine utilising high-throughput omics technologies including genomics, transcriptomics, metabolomics, proteomics, and epigenomics has gained increasing attention in the management of oncologic patients [57]. Recently, Sausen et al. have shown in patients with pancreatic adenocarcinoma with localized disease on imaging at initial diagnosis that circulating tumour DNA (ctDNA) was detected in $43 \%$ of cases, which predicted recurrence, poorer overall survival, and detected disease recurrence 6.5 months earlier than standard imaging [58].

The multianalyte gene transcript biomarker was developed for well-differentiated NEN and in our study provided information consistent with observations regarding clinical usefulness of liquid biopsies in other malignancies [23]. NETest was informative regarding the clinical diagnosis of a NEN, delineation of tumour burden reduction, response to treatment, and reflected disease status at follow-up. Filosso et al. demonstrated the clinical utility of NETest in a surgical series of patients with various types of lung malignancies, of them 28 with typical and atypical carcinoids, respectively. NETest accurately identified carcinoids, differentiated progressive from stable disease and verified completeness of surgical resection [30]. Genc et al. have shown in well-differentiated PanNEN that combining standard clinico-pathologic criteria and NETest results were approximately twice as effective as individual clinico-pathologic criteria alone since it positively correlated with recurrence in $\mathrm{R} 0$ resected patients [59]. A recent meta-analysis of NETest data by Oberg, et al. including ten original publications confirmed the diagnostic accuracy to be $95-96 \%$ and that chromogranin A which accounts for the standard tumour marker for NEN had no clinical utility [60]. In our own study, the NETest confirmed tumour mass reduction and could be used to stage patients post-surgery. As all had low-intermediate scores, there was no requirement for further therapeutic interventions. The NETest also functioned as an effective surrogate marker for stable disease and may have value in reducing imaging as has been noted [28].

Our study has some limitations. First, we have considered a strongly selected group of patients of whom all presented at initial diagnosis and were treatment naïve. All underwent surgery within 4 weeks after diagnostic completion and PRRT within 3 months following surgery, which does not necessarily reflect a real-world experience. In an ideal environment, cancer patients should be offered treatment within shortest time. Regretfully this is very frequently not the case in NEN patients. A recent survey showed that $50 \%$ of US patients reported being diagnosed with other conditions before receiving their NEN diagnosis which for $34 \%$ took 5 years or more and that there was delay in treatment after the diagnosis [61]. Similar experience has been reported in other countries [62]. Even when a NEN is assumed, it can take substantial time to complete the biochemical diagnostic process and secure a timely slot for somatostatin receptor-based PET/CT imaging. Starting with PRRT within 3 months following surgery may also be challenging due to: A) postoperative morbidity (e.g. anastomotic leakage or delayed /incomplete wound healing) precluding timely PRRT, B) not all centres have sufficient capacity to offer PRRT to a large number of patients [limitation of treatment sessions/week due to radiation protection regulations], C) depending upon local structures, shortage/delay in drug delivery may occur, and D) unforeseen technical issues may lead to ad hoc treatment session cancellation.

Second, we had no control group which would allow precise assessment of clinical significance of the concept presented in our study. Only a large, comparative treatment study would be able to determine "efficiency" of a modality in real word setting. This is unlikely to happen in NEN.

Third, although our data and experience of other groups suggest that NETest seems to be useful in the assessment of the adequacy of a treatment modality [31, 63, 64], a further long-term prospective study including a large number of consecutive patients is needed to establish the most accurate timing of 
post-interventional blood sampling as well as the metrics of the NETest in the prediction of residual or recurrent disease.

In conclusion, combining molecular imaging, surgery, and targeted molecular therapy provides a promising and safe approach for treatment of patients with well-differentiated metastasised SBNEN. The multianalyte biomarker NETest seems to be a promising tool for detection of neuroendocrine disease. It has the potential to define completeness of treatment and disease status. It may be used as a marker for disease stabilization. The efficacy of our approach provides a basis to support current efforts to establish the further development of precision treatment of oncologic patients.

\section{Acknowledgements}

\section{Funding}

This study was supported by a research grant to A.F. by Dr Heinz-Horst Deichmann Foundation. The funding source had no role in the design and conduct of the study; collection, management, analysis, and interpretation of the data; and preparation, review, or approval of the manuscript. Tissue samples were provided by the Imperial College Healthcare NHS Trust Tissue Bank. Other investigators may have received samples from these same tissues. The research was supported by the National Institute for Health Research (NIHR) Biomedical Research Centre based at Imperial College Healthcare NHS Trust and Imperial College London. The views expressed are those of the authors and not necessarily those of the NHS, the NIHR or the Department of Health.

\section{Author contributions}

Conception and design of the study: all authors contributed in this regard - it was a multinational study encompassing surgical, nuclear medical and radiological input, as well as analysis of novel multigene biomarkers.

Data acquisition and analysis: $\mathrm{AF}, \mathrm{AKC}, \mathrm{MK}$, IMM; Article writing: AF, AKC, MK, IMM led the writing with constructive input from all other authors; Final approval: All authors.

\section{Competing Interests}

MK is an employee of Wren Laboratories. IMM is a consultant to Wren Laboratories. A.F. has received educational grants and research grants from Novartis. A.F. acted as a speaker for Novartis and has received their speaker honorarium, for work not related to the present paper.

\section{References}

1 Norlén O, Stålberg P, Öberg K, Eriksson J, Hedberg J, Hessman O, et al. Long-term results of surgery for small intestinal neuroendocrine tumors at a tertiary referral center. World J Surg. 2012; 36(6):1419-31.

2 Saxena A, Chua TC, Perera M, Chu F, Morris DL. Surgical resection of hepatic metastases from neuroendocrine neoplasms: a systematic review. Surg Oncol. 2012; 21(3):e131-41.

3 Rindi G, Falconi M, Klersy C, Albarello L, Boninsegna L, Buchler MW, et al. TNM staging of Neoplasms of the Endocrine Pancreas: Results From a Large International Cohort Study. J Natl Cancer Inst. 2012; 104(10):764-77.

4 Falconi M, Eriksson B, Kaltsas G, Bartsch DKK, Capdevila J, Caplin M, et al. ENETS Consensus Guidelines Update for the Management of Patients with Functional Pancreatic Neuroendocrine Tumors and Non-Functional Pancreatic Neuroendocrine Tumors. Neuroendocrinology. 2016; 103(2):15371.

5 Niederle B, Pape UF, Costa F, Gross D, Kelestimur F, Knigge U, et al. ENETS consensus guidelines update for neuroendocrine neoplasms of the jejunum and ileum. Neuroendocrinology 2016; 103(2): 125-38.

6 Daskalakis K, Karakatsanis A, Hessman O, Stuart HC, Welin S, Tiensuu Janson E, et al. Association of a Prophylactic Surgical Approach to Stage IV Small Intestinal Neuroendocrine Tumors With Survival. JAMA Oncol. 2018; 4(2):183.

7 Citterio D, Pusceddu S, Facciorusso A, Coppa J, Milione M, Buzzoni R, et al. Primary tumour resection may improve survival in functional well-differentiated neuroendocrine tumours metastatic to the liver. Eur J Surg Oncol. 2017; 43(2):380-7.

8 Almond LM, Hodson J, Ford SJ, Gourevitch D, Roberts KJ, Shah T, et al. Role of palliative resection of the primary tumour in advanced pancreatic and small intestinal neuroendocrine tumours: A systematic review and meta-analysis. Eur J Surg Oncol. 2017; 43(10):1808-15.

9 Tsilimigras DI, Ntanasis-Stathopoulos I, Kostakis ID, Moris D, Schizas D, Cloyd JM, et al. Is Resection of Primary Midgut Neuroendocrine Tumors in Patients with Unresectable Metastatic Liver Disease Justified? A Systematic Review and Meta-Analysis. J Gastrointest Surg. 2019; 23(5):1044-1054.

10 Valkema R, Pauwels S, Kvols LK, Barone R, Jamar F, Bakker WH, et al. Survival and response after peptide receptor radionuclide therapy with [90Y-DOTA0,Tyr3]octreotide in patients with advanced gastroenteropancreatic neuroendocrine tumors. Semin Nucl Med. 2006; 36(2):147-56.

11 Strosberg J, El-Haddad G, Wolin E, Hendifar A, Yao J, Chasen B, et al. Phase 3 Trial of ${ }^{177}$ Lu-Dotatate for Midgut Neuroendocrine Tumors. N Engl J Med. 2017; 376(2):125-35.

12 Baum RP, Kulkarni HR. THERANOSTICS: From Molecular Imaging Using Ga-68 Labeled Tracers and PET/CT to Personalized Radionuclide Therapy The Bad Berka Experience. Theranostics. 2012; 2(5):437-47.

13 Arora G, Bandopadhyaya G. Paradigm shift in theranostics of neuroendocrine tumors: conceptual horizons of nanotechnology in nuclear medicine. Ann Nucl Med. 2018; 32(3):151-64.

14 Velikyan I. Molecular imaging and radiotherapy: Theranostics for personalized patient management. Theranostics. 2012; 2(5):424-6.

15 Crown A, Rocha FG, Raghu P, Lin B, Funk G, Alseidi A, et al. Impact of initial imaging with gallium-68 dotatate PET/CT on diagnosis and management of patients with neuroendocrine tumors. J Surg Oncol. 2020; 121(3):480-5.

16 Bodei L, Kidd M, Paganelli G, Grana CM, Drozdov I, Cremonesi M, et al. Long-term tolerability of PRRT in 807 patients with neuroendocrine tumours: the value and limitations of clinical factors. Eur J Nucl Med Mol Imaging. 2015; 42(1):5-19.

17 Brabander T, van der Zwan WA, Teunissen JJM, Kam BLR, Feelders RA, de Herder WW, et al. Long-Term Efficacy, Survival, and Safety of [177Lu-DOTA0,Tyr3]octreotate in Patients with Gastroenteropancreatic and Bronchial Neuroendocrine Tumors. Clin Cancer Res. 2017; 23(16):4617-24.

18 Pencharz D, Walker M, Yalchin M, Quigley AM, Caplin M, Toumpanakis C, et al. Early efficacy of and toxicity from lutetium-177-DOTATATE treatment in patients with progressive metastatic NET. Nucl Med Commun. 2017; 38(7):593-600

19 Imhof A, Brunner P, Marincek N, Briel M, Schindler C, Rasch H, et al. Response, survival, and long-term toxicity after therapy with the radiolabeled somatostatin analogue [90Y-DOTA]-TOC in metastasized neuroendocrine cancers. J Clin Oncol. 2011; 29(17):2416-23.

20 Sabet A, Dautzenberg K, Haslerud T, Aouf A, Sabet A, Simon B, et al Specific efficacy of peptide receptor radionuclide therapy with (177)Lu-octreotate in advanced neuroendocrine tumours of the small intestine. Eur J Nucl Med Mol Imaging 2015; 42(8):1238-46.

21 Sharma N, Naraev BG, Engelman EG, Zimmerman MB, Bushnell DL O'Dorisio TM, et al. Peptide receptor radionuclide therapy outcomes in a North American cohort with metastatic well-differentiated neuroendocrine tumors. Pancreas. 2017; 46(2):151-6.

22 Saravana-Bawan B, Bajwa A, Paterson J, McEwan AJB, McMullen TPW. Efficacy of 177Lu peptide receptor radionuclide therapy for the treatment of neuroendocrine tumors a meta-analysis. Clin Nucl Med. 2019; 44(9):719-27.

23 Siravegna G, Marsoni S, Siena S, Bardelli A. Integrating liquid biopsies into the management of cancer. Nat Rev Clin Oncol. 2017; 14(9):531-48. 
24 Pantel K, Alix-Panabières C. Liquid biopsy and minimal residual disease latest advances and implications for cure. Nat Rev Clin Oncol. 2019; 16(7):40924.

25 Modlin IM, Kidd M, Malczewska A, Drozdov I, Bodei L, Matar S, et al. The NETest. Endocrinol Metab Clin North Am. 2018; 47(3):485-504.

26 van Treijen MJC, Korse CM, van Leeuwaarde RS, Saveur LJ, Vriens MR, Verbeek WHM, et al. Blood Transcript Profiling for the Detection of Neuroendocrine Tumors: Results of a Large Independent Validation Study. Front Endocrinol (Lausanne). 2018; 9:740.

27 Al-Toubah TE, Cives M, Valone T, Blue K, Strosberg JR. Sensitivity and specificity of the NETest: A validation study. J Clin Oncol. 2019; 37(4 Suppl):222.

28 Liu E, Paulson S, Gulati A, Freudman J, Grosh W, Kafer S, et al. Assessment of NETest Clinical Utility in a U.S. Registry-Based Study. Oncologist. 2018; 24(6):783-790

29 Modlin IM, Frilling A, Salem RR, Alaimo D, Drymousis P, Wasan HS, et al. Blood measurement of neuroendocrine gene transcripts defines the effectiveness of operative resection and ablation strategies. Surgery. 2016; 159(1):336-47.

30 Filosso PL, Kidd M, Roffinella M, Lewczuk A, Chung K-M, Kolasinska-Cwikla A, et al. The utility of blood neuroendocrine gene transcript measurement in the diagnosis of bronchopulmonary neuroendocrine tumours and as a tool to evaluate surgical resection and disease progressiont. Eur J Cardio-Thoracic Surg. 2018; 53(3):631-9.

31 Ćwikła JB, Bodei L, Kolasinska-Ćwikła A, Sankowski A, Modlin IM, Kidd M. Circulating Transcript Analysis (NETest) in GEP-NETs Treated With Somatostatin Analogs Defines Therapy. J Clin Endocrinol Metab. 2015; 100(11):E1437-45

32 Deppen SA, Blume J, Bobbey AJ, Shah C, Graham MM, Lee P, et al. 68Ga-DOTATATE Compared with 111In-DTPA-Octreotide and Conventional Imaging for Pulmonary and Gastroenteropancreatic Neuroendocrine Tumors: A Systematic Review and Meta-Analysis. J Nucl Med. 2016; 57(6):872-8.

33 Baum RP, Kulkarni HR, Singh A, Kaemmerer D, Mueller D, Prasad V, et al. Results and adverse events of personalized peptide receptor radionuclide therapy with 90Yttrium and 177Lutetium in 1048 patients with neuroendocrine neoplasms. Oncotarget. 2018; 9(24):16932-50.

34 Young H, Baum R, Cremerius U, Herholz K, Hoekstra O, Lammertsma AA, et al. Measurement of clinical and subclinical tumour response using [18F]fluorodeoxyglucose and positron emission tomography: Review and 1999 EORTC recommendations. Eur J Cancer. 1999; 35(13):1773-82.

35 Eisenhauer EAA, Therasse P, Bogaerts J, Schwartz LHH, Sargent D, Ford R, et al. New response evaluation criteria in solid tumours: Revised RECIST guideline (version 1.1). Eur J Cancer. 2009; 45(2):228-47.

36 Ohrvall U, Eriksson B, Juhlin C, Karacagil S, Rastad J, Hellman P, et al. Method for dissection of mesenteric metastases in mid-gut carcinoid tumors. World J Surg. 2000; 24(11):1402-8.

37 Dindo D, Demartines N, Clavien P-A. Classification of surgical complications: a new proposal with evaluation in a cohort of 6336 patients and results of a survey. Ann Surg. 2004; 240(2):205-13.

38 Sadowski SM, Neychev V, Millo C, Shih J, Nilubol N, Herscovitch P, et al. Prospective study of 68Ga-DOTATATE positron emission tomography/computed tomography for detecting gastro-entero-pancreatic neuroendocrine tumors and unknown primary sites. J Clin Oncol. 2016; 34(6):588-97.

39 Hofman MS, Kong G, Neels OC, Eu P, Hong E, Hicks RJ. High management impact of Ga-68 DOTATATE (GaTate) PET/CT for imaging neuroendocrine and other somatostatin expressing tumours. J Med Imaging Radiat Oncol. 2012; 56(1):40-7.

40 Frilling A, Sotiropoulos GC, Radtke A, Malago M, Bockisch A, Kuehl H, et al. The impact of $68 \mathrm{Ga}$-DOTATOC positron emission tomography/computed tomography on the multimodal management of patients with neuroendocrine tumors. Ann Surg. 2010; 252(5):850-6.

41 Haugen BR. 2015 American Thyroid Association Management Guidelines for Adult Patients with Thyroid Nodules and Differentiated Thyroid Cancer: What is new and what has changed? Cancer. 2017; 123(3):372-81.

42 Sowa-Staszczak A, Pach D, Chrzan R, Trofimiuk M, Stefańska A, Tomaszuk $\mathrm{M}$, et al. Peptide receptor radionuclide therapy as a potential tool for neoadjuvant therapy in patients with inoperable neuroendocrine tumours (NETs). Eur J Nucl Med Mol Imaging. 2011; 38(9):1669-74.

43 van Vliet EI, van Eijck CH, de Krijger RR, Nieveen van Dijkum EJ, Teunissen JJ, Kam BL, et al. Neoadjuvant Treatment of Nonfunctioning Pancreatic Neuroendocrine Tumors with [177Lu-DOTA0,Tyr3]Octreotate. J Nucl Med. 2015; 56(11):1647-53

44 Stoeltzing O, Loss M, Huber E, Gross V, Eilles C, Mueller-Brand J, et al. Staged surgery with neoadjuvant 90Y-DOTATOC therapy for down-sizing synchronous bilobular hepatic metastases from a neuroendocrine pancreatic tumor. Langenbecks Arch Surg. 2010; 395(2):185-92.

45 Bertani E, Fazio N, Radice D, Zardini C, Grana C, Bodei L, et al. Resection of the Primary Tumor Followed by Peptide Receptor Radionuclide Therapy as Upfront Strategy for the Treatment of G1-G2 Pancreatic Neuroendocrine Tumors with Unresectable Liver Metastases. Ann Surg Oncol. 2016; 23(Suppl 5):981-9.

46 Kaemmerer D, Twrznik M, Kulkarni HR, Hörsch D, Sehner S, Baum RP, et al. Prior Resection of the Primary Tumor Prolongs Survival After Peptide
Receptor Radionuclide Therapy of Advanced Neuroendocrine Neoplasms. Ann Surg. 2019. DOI: 10.1097/SLA.0000000000003237

47 Partelli S, Bertani E, Bartolomei M, Perali C, Muffatti F, Grana CM, et al. Peptide receptor radionuclide therapy as neoadjuvant therapy for resectable or potentially resectable pancreatic neuroendocrine neoplasms. Surgery. 2017; 163(4):761-767.

48 Rinke A, Wittenberg M, Schade-Brittinger C, Aminossadati B, Ronicke E, Gress TM, et al. Placebo Controlled, Double Blind, Prospective, Randomized Study on the Effect of Octreotide LAR in the Control of Tumor Growth in Patients with Metastatic Neuroendocrine Midgut Tumors (PROMID): Results on Long Term Survival. Neuroendocrinology. 2016; 104(1):26-32.

49 Kwekkeboom DJ, Bakker WH, Kam BL, Teunissen JJM, Kooij PPM, Herder WW, et al. Treatment of patients with gastro-entero-pancreatic (GEP) tumours with the novel radiolabelled somatostatin analogue [177Lu-DOTA0,Tyr3]octreotate. Eur J Nucl Med Mol Imaging. 2003; 30(3):41722.

50 Figueiredo MN, Maggiori L, Gaujoux S, Couvelard A, Guedj N, Ruszniewski $\mathrm{P}$, et al. Surgery for small-bowel neuroendocrine tumors: is there any benefit of the laparoscopic approach? Surg Endosc. 2014; 28(5):1720-6.

51 Reissman P, Shmailov S, Grozinsky-Glasberg S, Gross DJ. Laparoscopic resection of primary midgut carcinoid tumors. Surg Endosc. 2013; 27(10):367882.

52 Clift AK, Faiz O, Al-Nahhas A, Bockisch A, Liedke MO, Schloericke E, et al. Role of Staging in Patients with Small Intestinal Neuroendocrine Tumours. J Gastrointest Surg. 2016; 20(1):180-8.

53 Lardière-Deguelte S, De Mestier L, Appéré F, Vullierme MP, Zappa M, Hoeffel $\mathrm{C}$, et al. Toward a preoperative classification of lymph node metastases in patients with small intestinal neuroendocrine tumors in the era of intestinal-sparing surgery. Neuroendocrinology. 2016; 103(5):552-9.

54 Pasquer A, Walter T, Rousset P, Hervieu V, Forestier J, Lombard-Bohas C, et al. Lymphadenectomy during Small Bowel Neuroendocrine Tumor Surgery: The Concept of Skip Metastases. Ann Surg Oncol. 2016; 23(S5):804-8.

55 Kaemmerer D, Prasad V, Daffner W, Haugvik SP, Senftleben S, Baum RP, et al. Radioguided surgery in neuroendocrine tumors using Ga-68-labeled somatostatin analogs: A pilot study. Clin Nucl Med. 2012; 37(2):142-7.

56 El Lakis M, Gianakou A, Nockel P, Wiseman D, Tirosh A, Quezado MA, et al. Radioguided Surgery with Gallium 68 Dotatate for Patients with Neuroendocrine Tumors. JAMA Surg. 2019; 154(1):40-5.

57 Sandhu C, Qureshi A, Emili A. Panomics for Precision Medicine. Trends Mol Med. 2018; 24(1):85-101.

58 Sausen M, Phallen J, Adleff V, Jones S, Leary RJ, Barrett MT, et al. Clinical implications of genomic alterations in the tumour and circulation of pancreatic cancer patients. Nat Commun. 2015; 6:7686.

59 Genç CG, Jilesen APJ, Nieveen van Dijkum EJM, Klümpen H-J, van Eijck CHJ, Drozdov I, et al. Measurement of circulating transcript levels (NETest) to detect disease recurrence and improve follow-up after curative surgical resection of well-differentiated pancreatic neuroendocrine tumors. J Surg Oncol. 2018; 118(1):37-48.

60 Öberg K, Califano A, Strosberg JR, Ma S, Pape U, Bodei L, et al. A meta-analysis of the accuracy of a neuroendocrine tumor mRNA genomic biomarker (NETest) in blood. Ann Oncol. 2020; 31(2):202-12.

61 Wolin EM, Leyden J, Goldstein G, Kolarova T, Hollander R, Warner RRP. Patient-reported experience of diagnosis, management, and burden of neuroendocrine tumors: Results from a large patient survey in the United States. Pancreas. 2017; 46(5):639-47.

62 Basuroy R, Bouvier C, Ramage JK, Sissons M, Srirajaskanthan R. Delays and routes to diagnosis of neuroendocrine tumours. BMC Cancer. 2018; 18(1). DOI: 10.1186/s12885-018-5057-3

63 Bodei L, Kidd MS, Singh A, van der Zwan WA, Severi S, Drozdov IA, et al. PRRT genomic signature in blood for prediction of 177Lu-octreotate efficacy. Eur J Nucl Med Mol Imaging. 2018; 45(7):1155-69.

64 Partelli S, Andreasi V, Muffatti F, Schiavo Lena M, Falconi M. Circulating Neuroendocrine Gene Transcripts (NETest): A Postoperative Strategy for Early Identification of the Efficacy of Radical Surgery for Pancreatic Neuroendocrine Tumors. Ann Surg Oncol. 2020; DOI: 10.1245 /s10434-020-08425-6 